\section{Genome Resource of Podosphaera xanthii, the Host-Specific Fungal Pathogen That Causes Cucurbit Powdery Mildew}

\author{
Seunghwan Kim, ${ }^{1}$ Sathiyamoorthy Subramaniyam, ${ }^{2}$ Myunghee Jung, ${ }^{2}$ Eun-A Oh, ${ }^{1}$ Tae Ho Kim, ${ }^{1}$ \\ and Jeong-Gu Kim ${ }^{1, \dagger}$ \\ ${ }^{1}$ Genomics Division, National Institute of Agricultural Science, RDA, 370, Nongsaengmyeong-ro, \\ Jeonju-si 54874, Republic of Korea \\ ${ }^{2}$ Research and Development Center, Insilicogen Inc., Yongin-si 16954, Gyeonggi-do, Republic of Korea
}

\begin{abstract}
Approximately 33 types of commonly consumed fruits and vegetables are members of the family Cucurbitaceae, making it an important crop family worldwide. However, pathogen resistance to pesticides and fungicides has become a growing problem in cultivation practices. The identification of the effector proteins in each unique fungus-host pair would help toward the development of strategies for preventing the infection of important crops. In this study, we characterized the genome of Podosphaera xanthii, the fungal pathogen that causes powdery mildew disease in cucurbitaceous plants. A first-draft genome of $209.08 \mathrm{MB}$ was assembled and compared with those of 25 other fungal pathogens, particularly for identifying candidate secreted effector proteins. This draft genome can serve as a valuable resource for future genomic and proteomic studies of $P$. xanthii and its host-specific pathogenesis.
\end{abstract}

\section{Genome Announcement}

Powdery mildew disease (PMD) is a plant disease that is caused by members of the fungal family Erysiphaceae (Cook, 2012), of which there are currently 724 known species across 27 genera (NCBI Taxonomy Database; accessed April 2020). These obligate parasites exist mainly on the plant leaves, where they attach to the surfaces via their haustoria and in the form of conidiophores (Polonio et al. 2019b). They are airborne and highly resistant to cold and drought environments (De Miccolis Angelini et al. 2019). Since $300 \mathrm{BCE}, \mathrm{PMD}$ has been recorded as a major disease of a large number of plants (Wu et al. 2018). Although many PMD-causing pathogens have identical morphological characteristics, the measures adopted for their control (e.g., spraying of biocontrollers and fungicides) are not unique and are, in fact, mostly ineffective. A proposed alternative approach is to identify the effector proteins from each fungus-host pair, which would help toward the development of targeted biocontrollers or

\section{${ }^{\dagger}$ Corresponding author: J.-G. Kim; jkim5aug@korea.kr}

S. Kim and S. Subramaniyam contributed equally as first authors.

Competing interests: Authors S. Subramaniyam and M. Jung were employed by the company Insilicogen Inc. The remaining authors declare that the research was conducted in the absence of any commercial or financial relationships that could be construed as a potential conflict of interest.

*The $e$-Xtra logo stands for "electronic extra" and indicates that a supplementary file, supplementary figures, and supplementary tables are published online.

Accepted for publication 1 December 2020.
$e-\mathbf{X t r a}^{*}$

Funding

This work was supported by the Agricultural Agenda Program (PJ01256301), Rural Development Administration, Republic of Korea.

\section{Keywords}

Cucurbitaceae, Erysiphaceae, fungus-plant interactions, genome, genomics, metabolomics, pathogen, Podosphaera xanthii, proteomics 
Table 1. Summary of genome assemblies and gene annotations

\begin{tabular}{lc} 
Assemblies and annotations & Statistics \\
Assembly & \\
Contigs & 1,112 \\
Total bases & $209,067,775$ \\
Average length & $188,010.59$ \\
Minimum length & 19,987 \\
Maximum length & $2,325,138$ \\
$N_{50}$ & 581,650 \\
$N$ (\%) & 0.00 \\
GC (\%) & 44.25 \\
Gene & \\
Genes & 12,834 \\
Average gene length & $2,418.79$ \\
Average exon length & 425.23 \\
Repeat elements & $132,699,478(63.41 \%)$ \\
Gene coverage & $14.83 \%$ \\
BUSCO & $10,461(81.51 \%)$ \\
Annotations & $7,375(57.46 \%)$ \\
Blast-hits & \\
Kyoto Encyclopedia of Genes and Genomes & $8,529(66.46 \%)$ \\
(KEGG) & $2,373(18.49 \%)$ \\
Gene Ontology (GO) & 455 \\
No-hits & 690 \\
Candidate secrete effector proteins (CSEP) & 169 \\
Secreted proteins (SP) & $37.37, \mathrm{n}: 758$ \\
Nonclassical secreted proteins (NCSP) & 519 \\
Cytochrome (CYP) & \\
Carbohydrate-active enzymes (CAZY) & \\
\hline
\end{tabular}

fungicides (Martínez-Cruz et al. 2018; Polonio et al. 2019a). To accelerate this approach, elucidation of the pathogenesis of PMD at the molecular level would be the primary goal, of which the minimum requirement is the reference genomes of both the hosts and the pathogens. Although the genomes of a few PMD-causing fungi have been sequenced and used to aid the identification and annotation of some candidate secreted effector proteins (CSEPs) through functional assays (Martínez-Cruz et al. 2018), most of the pathogens that cause this disease have not been genomically characterized (Bindschedler et al. 2016). In this study, we sequenced the genome of Podosphaera xanthii, a highly infectious PMD-causing pathogen. Several studies have generated genetic resources for this fungal species before, such as features of the transcriptome from epiphytic fungal material (Vela-Corcía et al. 2016), the haustorium (Polonio et al. 2019b), the progenies of sexual mating (De Miccolis Angelini et al. 2019), the genetic diversity among $P$. xanthii races assessed by genotype-based sequencing (Hong et al. 2018; Xiang et al. 2020), and the mitochondrial genome (Kim et al. 2019). However, most of these studies gained partial knowledge only owing to the lack of a full reference genome. To the best of our knowledge, the genome assembled in this study has better contiguity $\mathrm{N}_{50}$ score compared with the first available draft genome of $P$. xanthii (Polonio et al. in press).

\section{Preliminary Genome Report}

Our dataset includes both long- and short-read sequences from the DNA and mRNA molecules of $P$. xanthii (Supplementary Table S1). The detailed methods used to assemble and annotate the genome are presented as a workflow in Supplementary Figure S1. To ensure that the sequenced molecules were from $P$. xanthii, three contamination checks were conducted: host contamination (Cucumis sativus), bacterial contaminations, and other fungal cocontaminations. The genome of $P$. xanthii was estimated to be $253.13 \mathrm{MB}$ in size, as determined with the $k$-mer major peak method using the contamination- and mitochondriafree short reads (Supplementary Fig. S2A). Of the assembled genome, 209.07 MB was obtained from error-corrected long-read sequences (38.9 GB) using the haplotype-aware assembler FALCON-Unzip v0.30 (Table 1). The assembled contigs were checked for contamination using the candidate selected from the short-read contamination removal process (Supplementary Table S2 and S3). In this assembly, $133 \mathrm{MB}(63.41 \%)$ of the full 
genome was predicted to be repeats, most of which were long terminal repeats. The genome carried 12,834 genes in total, according to the predictions of three modules: an evidencebased gene modeler (EvidenceModeler), an ab initio gene modeler, and a consensus gene modeler (Table 1). Among those genes, 10,461 (81.51\%) were homologous with genes of other genomes in the GenBank NR nucleotide database (Table 1). The assembled genome was assessed for two different quality metrics: $\mathrm{N}_{50}(5.8 \mathrm{MB})$ for contiguity and BUSCO $(98.3 \%$ for the Fungi dataset) for gene completeness (Supplementary Fig. S3 for other BUSCO datasets). Finally, genomes were assessed for identity with the internal transcribed spacer (ITS) from the assembled genome, because the in vitro culture of $P$. xanthii is difficult without the host (Martínez-Cruz et al. 2018). The complete ITS fragments present in the genome (Supplementary Table S4) were identical to MT242593.1, a GenBank reference of the $P$. xanthii ribosomal RNA gene. Additionally, the BLAST score $(E-v a l u e=0.0)$ also confirmed the genome identity as $P$. xanthii. Finally, the $P$. xanthii proteome was compared with those of 25 other fungal pathogens that cause various diseases in different crops (Supplementary Table S5). This comparative genome analysis provided an overview of the CSEPs and other multifamily proteins (e.g., cytochromes and carbohydrate-active enzymes) in this fungus through the perspective of gene gain-of-function and loss-of-function analyses (Table 1; Supplementary Fig. S2C), along with its evolutionary relationships. Furthermore, the genome size and proteome revealed that the $P$. xanthii genome is the second largest of the PMDcausative pathogens (Supplementary Fig. S2B). In addition, the transcriptome libraries from this study and entries in the Sequence Read Archive (SRA) database were mapped to this draft genome (Supplementary Table S6).

\section{Dataset Information to the User}

The complete sequences generated in this study were deposited in the SRA repository under the accession PRJNA590048. The assembled contigs and the annotation files (coding sequences, gff, repeats, and proteins) are available in the figshare repository with all the annotations details in the Readme file. The contig assembly of this draft genome was submitted to the NCBI-Assembly under the accession JAAAXZ000000000. The materials and methods are given in Supplementary File S1.

\section{Author-Recommended Internet Resources}

Figshare repository: https://figshare.com/articles/dataset/P_xanthii_genome_project/12292841

\section{Literature Cited}

Bindschedler, L. V., Panstruga, R., and Spanu, P. D. 2016. Mildew-omics: How global analyses aid the understanding of life and evolution of powdery mildews. Front. Plant Sci. 7:123.

Braun, U., and Cook, R. T. A. 2012. Taxonomic Manual of the Erysiphales (Powdery Mildews). CBS Biodiversity Series 11. CBS-KNAW Fungal Biodiversity Centre, Utrecht, The Netherlands.

De Miccolis Angelini, R. M., Pollastro, S., Rotondo, P. R., Laguardia, C., Abate, D., Rotolo, C., and Faretra, F. 2019. Transcriptome sequence resource for the cucurbit powdery mildew pathogen Podosphaera xanthii. Sci. Data 6:95.

Hong, Y.-J., Hossain, M. R., Kim, H.-T., Park, J.-I., and Nou, I.-S. 2018. Identification of two new races of Podosphaera xanthii causing powdery mildew in melon in South Korea. Plant Pathol. J. 34:182-190.

Kim, S., Jung, M., Oh, E. A., Kim, T. H., and Kim, J.-G. 2019. Mitochondrial genome of the Podosphaera xanthii: A plant pathogen causes powdery mildew in cucurbits. Mitochondrial DNA Part B 4:4172-4173.

Martínez-Cruz, J., Romero, D., de la Torre, F. N., Fernández-Ortuño, D., Torés, J. A., de Vicente, A., and Pérez-García, A. 2018. The functional characterization of Podosphaera xanthii candidate effector genes reveals novel target functions for fungal pathogenicity. Mol. Plant-Microbe Interact. 31:914-931.

Polonio, Á., Díaz-Martínez, L., Fernández-Ortuño, D., de Vicente, A., Romero, D. F., López-Ruiz, F., and Perez-Garcia, A. A hybrid genome assembly resource for
Podosphaera xanthii, the main causal agent of powdery mildew disease in cucurbits. Mol. Plant-Microbe Interact. In press. doi.org/10.1094/MPMI-08-200237-A

Polonio, Á., Pineda, M., Bautista, R., Martínez-Cruz, J., Pérez-Bueno, M. L., Barón, M., and Pérez-Garcia, A. 2019a. RNA-seq analysis and fluorescence imaging of melon powdery mildew disease reveal an orchestrated reprogramming of host physiology. Sci. Rep. 9:7978.

Polonio, Á., Seoane, P., Claros, M. G., and Pérez-Garcia, A. 2019b. The haustorial transcriptome of the cucurbit pathogen Podosphaera xanthii reveals new insights into the biotrophy and pathogenesis of powdery mildew fungi. BMC Genomics 20:543.

Vela-Corcia, D., Bautista, R., de Vicente, A., Spanu, P. D., and Pérez-García, A. 2016. De novo analysis of the epiphytic transcriptome of the cucurbit powdery mildew fungus Podosphaera xanthii and identification of candidate secreted effector proteins. PLoS One 11:e0163379.

Wu, Y., Ma, X., Pan, Z., Kale, S. D., Song, Y., King, H., Zhang, Q., Presley, C., Deng, X., Wei, C.-I., and Xiao, S. 2018. Comparative genome analyses reveal sequence features reflecting distinct modes of host-adaptation between dicot and monocot powdery mildew. BMC Genomics 19:705.

Xiang, Y., Miller, A. N., McGrath, M., and Babadoost, M. 2020. Genotyping-bysequencing for analysis of the genetic variation of Podosphaera xanthii, incitant of cucurbit powdery mildew. Plant Dis. 104:951-957. 